\title{
Cryptographic Key Exchange Authentication using Two Servers PAKE
}

\author{
G.K. Karthika, Raju Dara, Yamini Devi. N
}

\begin{abstract}
The key trade procedure is well thought-out significant fractions of cryptographic method towards defend protected end-to-end communications. All existing techniques need two servers to be active to authenticate but this technique can authenticate even one server is up and other server is down due to attack as active server authenticate user by taking his parts. In this paper, design an idea Password-authenticated key exchange (PAKE) method to verify clients by utilizing two servers, first client secret phrase will be splitted into two sections and afterward mystery key will be produced for each part and after that by utilizing key and splitted secret phrase will be encoded utilizing Elgamal Encryption. Each encoded part and key will be send to every server. Every server will have its very own key and in the event that aggressor traded off one server, at that point he won't ready to login till he got client information of second server, by utilizing this strategy no assailant can bargain the two servers.
\end{abstract}

Keywords: Cryptography, ElGamal Encryption, Password Authenticated Key Exchange (PAKE..

\section{INTRODUCTION}

These days, code word are regularly utilized by individuals during a sign in procedure so as to have power over way in to verified PC running system, phones, satellite TV decoders, robotized teller machinery, etc. APC client possibly will necessitate code word intended for some reasons: signing within towards PC explanation, recovering email from servers, attainment on the way to programs, record, scheme, web locales, along with in spite of pay particular attention on the web. Prior secret key based confirmation frameworks transmitted cryptographic hash of the secret key more an open conduit which construct the confusion esteem open on the way to an aggressor. At the point while this be completed, as well as it be normal, the aggressor be able to effort disconnected, quickly testing potential secret word alongside the genuine secret word's confusion esteem. Learn contain reliably demonstrated so as to an enormous portion of client picked passwords are promptly speculated consequently. For instance, as indicated by Bruce Schneier, looking at information from a 2006phishing assault, 55 percent of MySpace code words prospective break capable within 8 hours utilizing an industrially accessible Secret key Recovery Toolkit fit for testing 200,000passwords every second in 2006. Ongoing exploration propels in secret word based

Revised Version Manuscript Received on August 14, 2019.

Ms. G.K. Karthika, Asst. Professor, Dept., of Information Technology, Vignana Bharathi Institute of Technology, Hyderabad, Telangana, India. (E-Mail: kalpeesri@gmail.com)

Dr. Raju Dara Professor, Dept., of Computer Science \& Engineering, Vignana Bharathi Institute of Technology, Hyderabad, Telangana, India

(E-Mailrajurdara@gmail.com)

Ms. Yamini Devi.N Dept., of Computer Science \& Engineering, Vignana Bharathi Institute of Technology, Hyderabad, Telangana, India

(E-Mail: kalpeesri@gmail.com) confirmation have permitted a customer as well as a attendant commonly towards validate by way of a secret phrase along with in the interim on the way to build awake a cryptographic input meant for safe interchanges subsequent to confirmation. All within all, present answers designed for code word support confirmation pursue two representations. The principal representation, known as PKI-based form, accept so as to the customer maintains the server open key notwithstanding distributes a secret word through the server. Within these surroundings, the customers know how to send the secret word towards the server next to open key encryption. Gonget al. be the primary towards exhibit this sort of verification conventions among heuristic impervious near disconnected lexicon assaults, Halevi as well as Krawczyk be the initial towards give proper description also thorough evidences of safety meant for PKI-based design. The subsequent design was known as secret phrase just method. Bellovin along with Merritt be the primary in the direction of believe confirmation dependent lying on secret word just, moreover presented a position of alleged "scrambled key in trade" conventions, anywhere the secret phrase be utilized because a mystery key in to scramble arbitrary information meant for key in trade reason. proper form of safety designed for the secret phrase just verification be initial specified freely through Bellare et al. as well as Boyko et al.Katz et al. be the first towards provide secret key as it we reconfirmation convention which is both reasonable along with verifiably safe beneath criterion cryptographic presumption. In view of the character based encryption method Yi et al. proposed a character pedestal form anywhere the customer wants towards recollect the secret key as it were while the server keeps secret key notwithstanding private keys identified with its character. Within this background, the customer be able to encode the secret key dependent resting on the personality of the attendant. This reproduction be stuck between the PKI-based as well as the secret key as it we remodels. A commonplace convention for secret key based validationexpect a solitary server stores every one of the passwords important to validate customers. On the off chance that the server be undermined, owing towards, designed for instance, slash, otherwise introducing a "Trojan pony," or else still within assault, client secret words put away inside the server be revealed. in the direction of tackle this subject, two-server secret phrase pedestal confirmation conventions were presented, where two servers collaborate to confirm a customer based on secret key and in the event that one server is undermined, the assailant still 
hypocrisy claim towards exist the client through the data as of the traded off server.

We designed Two Server Password-authenticated key exchanges (PAKE) are where servers collaborate to validate a client and in the event that one server is undermined, the aggressor regardless can't claim to be the shopper with the data from the traded off server. The present answers for 2-server PAKE are either symmetric in the vibe those servers similarly add to the verification or uneven inside the vibe that one server verifies the client with the help of any other server. We're right here by means of looking to awareness on the symmetric answer for 2-server PAKE, in which the client can set up one of a kind cryptographic key by way of the 2 servers, separately. Our convention keeps running in parallel and is additional green than present symmetric - server PAKE convention, or considerably more green than existing awry two-server PAKE conventions in expressions of equivalent calculation. Similarly to that a nonce might be generated throughout the length of authentication and this may act as a timer. If the timer does no longer expire with in the period restrict, the authentication process might be achieved in the restriction which presents protection to replay assaults. A symmetric server PAKE convention can keep running in parallel and builds up secret session keys between the buyer and servers, separately. In the event that one of the servers closes down because of the disavowal of-administration ambush, some other server can keep on giving administrations to validated customers. Wellbeing examination has demonstrated that our convention is secure contrary to every inactive and vivacious assault on the off chance that that one server is undermined. Execution assessment has demonstrated that our convention is extra productive than current symmetric and awry - server PAKE conventions regarding parallel calculation.

\section{METHODOLOGY \& RESULTS}

In our framework, there exist two servers $\mathrm{S} 1$ along with $\mathrm{S} 2$ along with a meeting of clients. The 2 servers coordinate to confirm customers along with supply administrations to validated clients. Before confirmation, each purchaser $\mathrm{C}$ selections a secret word $p w_{C}$ in addition to creates the name of the game key verification in sequence $A u t h_{C}^{(1)}$ along with $A u t h_{C}^{(2)}$ intended for S1 as well as S2, separately, with the end aim that no one can decide the name of the secret keypw from $A u t h_{C}^{(1)}$ or else $A u t h_{C}^{(2)}$ except if S1 along with S2 plot. The client sends $A u t h_{C}^{(1)}$ along with $A u t h_{C}^{(2)}$ to S1 along with $\mathrm{S} 2$, precise, thru diverse at ease channels at some stage in the patron enrolment. From that factor onward, the purchaser recalls the secret phrase just, along with the two servers maintain the name of the game key verification records. Like every single existing answer for 2-server PAKE, we receive the 2 servers in no way plot to uncover the name of the game word of the purchaser. At the point when the 2 servers coordinate to validate a client $\mathrm{C}$, we expect that the consumer $\mathrm{C}$ can communicate a message to each of $\mathrm{S} 1$ along with $\mathrm{S} 2$ all of the even as, but strain that we do not take delivery of a communicate channel along with, specially, an aggressor can carry diverse messages to the 2 servers or decline to convey a message to a server. In our convention,

the client along with the 2 servers convey thru an open channel which might be spied, postponed, replayed, along with even altered by means of an aggressor. Our conference is symmetric if servers further upload to the confirmation as a ways as calculation along with correspondence.

\section{ElGamal Encryption:}

In cryptography, the ElGamal encryption structure be a lopsided key in encryption computation intended for release key in cryptography. The ElGamal encryption plan be created by ElGamalin 1985 based scheduled Diffie-Hellman key trade convention. It comprise of key age, encryption, along with unscrambling computations:

Key Generation: going on information a safety constraint $\mathrm{k}$, it distributes a cyclical gathering $\mathrm{G}$ of huge prime request $\mathrm{q}$ by means of a producer $\mathrm{g}$. At that point it picks a decoding key $\mathrm{x}$ arbitrarily on or after $Z_{q}^{*}$ along with processes an encryption key $\mathrm{y}=g^{x}$.

Encryption: On inputs a communication $m \in G$ along with the encryption key $\mathrm{y}$, it decides a digit $\mathrm{r}$ along withomlyfrom $Z_{q}^{*} \quad$ along with outputs a ciphertext $\mathrm{C}=\mathrm{E}(\mathrm{m}, \mathrm{y})=(\mathrm{A}, \mathrm{B})=\left(g^{r}, m, y^{r}\right)$.

Decryption: On data sources a ciphertext $(\mathrm{A}, \mathrm{B})$, along with the decoding key $\mathrm{x}$, it yields the plaintext $\mathrm{m}=\mathrm{D}(\mathrm{C}, \mathrm{x})=\mathrm{B} / A^{x}$.

ElGamal encryption plan is a probabilistic encryption plot. On the off chance that encoding a similar significance by means of ElGamal encryption conspires a few times, it determination, when all is said in done, yield diverse ciphertexts. Tsioun is along with Yung demonstrated ElGamal encryption plan to exist semantically protected under the DDH supposition.

\section{RESULTS ALONG WITH DISCUSSION}

In this area, we examine the presentation of our convention what's more, contrast our convention along with existing conventions for two server secret phrase just validation along with key trade. In database also we can see password save in encrypted format.

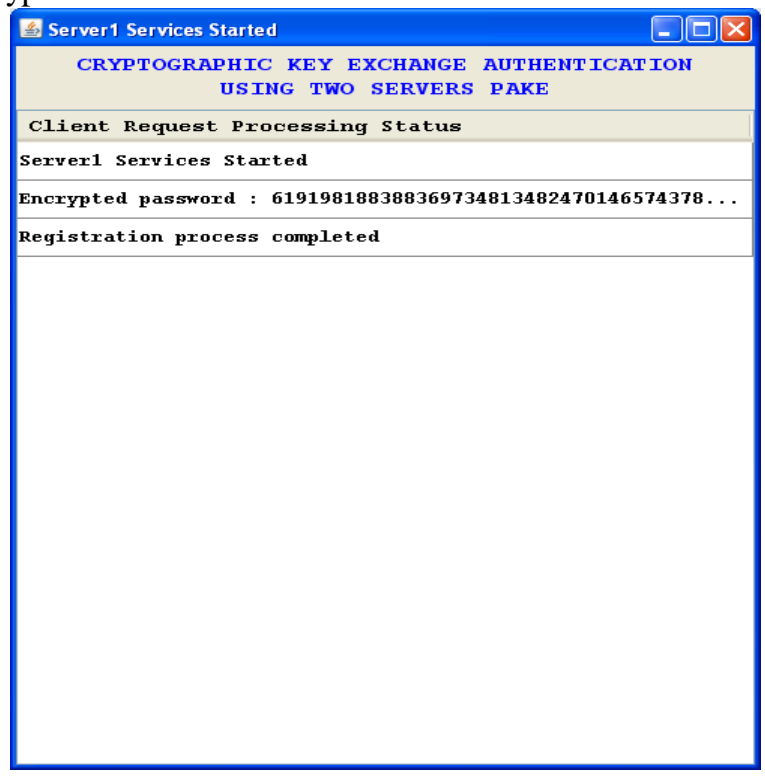




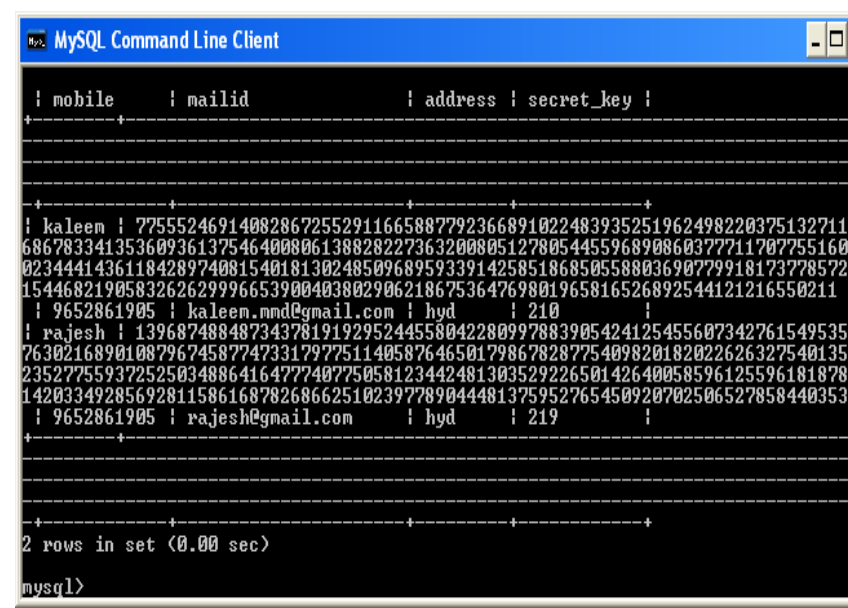

Now click on login button to authenticate user with two servers. Then in that screen, we can see user login successfully by using two servers. Now close black console of one server along with then still we can login. This means even more server down then second server can able to login user or provide services to the user. See below server screen for password.

\begin{tabular}{l} 
Server1 Services Started \\
CRYPTOGRAPHIC KEY EXCHANGE AUTHENTICATION \\
US ING TWO SERVERS PAKE \\
Client Request Processing Status \\
Server1 Services Started \\
Encrypted password : 619198188388369734813482470146574378... \\
\hline Registration process completed \\
Encrypted password : $619198188388369734813482470146574378 \ldots$ \\
\hline success \\
\hline Encrypted password : $955692818058380052074572830342100045 \ldots$ \\
\hline fail \\
\hline Encrypted password : $619198188388369734813482470146574378 \ldots$ \\
\hline success \\
\hline
\end{tabular}

\section{CONCLUSION}

In this paper, we planned a concept to authenticate users by using two servers, first user password will be splitted into two parts along with then secret key will be generated for each part along with then by using key along with splitted password will be encrypted using Elgamal Encryption. Each encrypted part along with key will be send to each server. Each server will have its own key along with if attaScker compromised one server then he won't able to login till he got user data of second server, by using this technique no attacker can compromise both servers.

\section{REFERENCES}

1. Alix. "Predictive estimation of protein linear epitopes by using the program PEOPLE". Vaccine, 18:311-4, 1999.

2. Argos, P., Rossmann, M.G., Grau, U.M., Zuber, H., Frand, G., \& Tratschin, J.D. (1979) Biochemistry 18,
$5698-5703$

3. Atassi, M. Z. \& Lee, C. L. (1978) Biochemistry 171, 429-434.

4. Atassi, M. Z. (1975) Immunochemistry 12, 423438.

5. Atsushi Ikai(1980).., "Thermostability and Aliphatic Index of Globular proteins".., Biochem. 88, 1895-1898 (1980)

6. B. Peters, J. Sidney, P. Bourne, H. Bui, S. Buus, G. Doh, W. Fleri, M. Kronenberg, R. Kubo, O. Lund, et al. "The Immune Epitope Database and Analysis Resource: From Vision to Blueprint”. PLoS Biology, 3:e91, 2005.

7. Bachmair.A., Finley,D. and Varshavsky.A. (1986) Science, 234, 179-186.

8. Baranyi L, Campbell W, Ohshima K, Fujimoto S, Boros $\mathrm{M}$, Okada H. "The antisense homology box. a new motif within proteins that encodes biologically active peptides". Nat. Med 1995, 1, pp. 894901.

9. Barlow,D.J., Edwards, M.S., and Thornton,J.M., 1986 "Continuous and discontinuous protein antigenic determinants'. Nature, Vol 322, pp747-748.

10. Berchanski A, Shapira B, Eisenstein M. "Hydrophobic complementarity in protein protein docking". Proteins 2004, 56, pp. 130142.

11. Chen. J, H. Liu, J. Yang, and K. Chou." Prediction of linear B-cell epitopes using amino acid pair antigenicity scale". Amino Acids, 33:423-428, 2007.

12. Chou PY, Fasman GD. 1974. "Conformational parameters for amino acids in helical, \&sheet and random coil regions calculated from proteins". Eiochemistry 13:211-223

13. Clements JD, Martin RE. "Identification of novel membrane proteins by searching for patterns in hydropathy profiles". Eur. J. Biochem 2002, 269, pp. 21012107.

14. Creighton.T.E. (1988) BioEssays, 8, 57-63.

15. Curr ..,'Design of synthetic peptides for diagnostics", Protein Pept Sci, 4(4):253-260, 2003.

16. D. Flower. "Immunoinformatics: "Predicting immunogenicity in silico". Quantum distributor, 1st edition, 2007

17. T. El Gamal, A public key cryptosystem along with a signature scheme based on discrete logarithms, IEEE Trans. Inform. Theory 31 (1985) 469-472.

18. W. Ford, B.S. Kaliski, Server-assisted generation of a strong secret from a password, in: Proc. 5th IEEE Intl. Workshop on Enterprise Security, 2000.

19. R. Gennaro, Y. Lindell, A framework for password-based authenticated key exchange, ACM Trans. Inf. Syst. Secur. 9 (2) (2006) 181-234.

20. O. Goldreich, Y. Lindell, Session-key generation using human passwords only, J. Cryptology 19 (3) (2006) 241-340, preliminary version in Crypto 2001.

21. L. Gong, T.M.A. Lomas, R.M. Needham, J.H. Saltzer, Protecting poorly-chosen secrets from guessing attacks, IEEE J. Sel. Areas Commun. 11 (5) (1993)648-656.

22. S. Halevi, H. Krawczyk, Public-key cryptography along with password protocols, ACM Trans. Inf. Syst. Secur. 2 (3) (1999) 230-268.

23. D. Jablon, Strong password-only authenticated key exchange, ACM Comput. Commun. Rev. 26 (5) (1996) $5-20$.

24. D. Jablon, Password authentication using multiple servers, in: RSA Cryptographers' Track 2001, in: Lecture Notes in Comput. Sci., vol. 2020, Springer-Verlag, 2001, pp. 344-360. 
25. S. Jiang, G. Gong, Password based key exchange with mutual authentication, Workshop on Selected Areas of Cryptography (SAC), 2004

\section{ACKNOWLEDGEMENT}

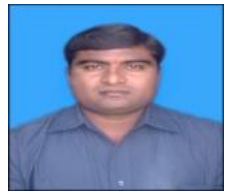

Dr. Raju Dara is a professor of Computer Science along with Engineering Department at Vignana Bharathi Institute of Technology, Hyderabad. He has 16 years of teaching experience for Graduate along with Post Graduate engineering courses. His current research interests are Data Warehousing, Image Processing, along with Network Security. He published 30 research papers in international journals as well as international conferences. I will be grateful to the University Grants Commission for the certain financial aid, along with thankful to the FIST laboratories for providing required computational facilities at the institution, eventually, I extend the deep sense of gratitude to the management, principal, director of R\&D of Vignana Bharathi Institute of Technology for supporting in accomplishment of the minor research project on time lucratively. 